\title{
A theoretical analysis for gigabit/second pulse code modulation of semiconductor lasers
}

Danielsen, Magnus

Published in:

I E E E Journal of Quantum Electronics

Publication date:

1976

Document Version

Publisher's PDF, also known as Version of record

Link back to DTU Orbit

Citation (APA):

Danielsen, M. (1976). A theoretical analysis for gigabit/second pulse code modulation of semiconductor lasers. I E E E Journal of Quantum Electronics, 12(11), 657-660.

\section{General rights}

Copyright and moral rights for the publications made accessible in the public portal are retained by the authors and/or other copyright owners and it is a condition of accessing publications that users recognise and abide by the legal requirements associated with these rights.

- Users may download and print one copy of any publication from the public portal for the purpose of private study or research.

- You may not further distribute the material or use it for any profit-making activity or commercial gain

- You may freely distribute the URL identifying the publication in the public portal

If you believe that this document breaches copyright please contact us providing details, and we will remove access to the work immediately and investigate your claim. 


\title{
A Theoretical Analysis for Gigabit/Second Pulse Code Modulation of Semiconductor Lasers
}

\author{
MAGNUS DANIELSEN, MEMBER, IEEE
}

\begin{abstract}
Investigation of the rate equations of a semiconductor laser suggests that bit rates of 3-4 Gbit/s can be achieved. Delay, ringing transients, and charge-storage effects can be removed by adjusting the dc-bias current and the peak and width of the current pulse to values prescribed by simple analy tical expressions. Also, simple approximate formulas for the light pulse maximum, width, delay, and integrated values are given.
\end{abstract}

\section{INTRODUCTION}

$\mathbf{P}$ ULSE modulation of a semiconductor laser presents three problems [1]-[5], when high bit rates are required: a time delay between the applied current pulse and the light pulse, ringing transients, and the charge-storage effect of preceding pulses on the light pulse form and magnitude.

This work presents a pulse modulation model which avoids these disadvantages. A dc-bias current near threshold reduces the delay. The first spike of the ringings is suggested to be exploited as a light pulse. This is done by switching off the current pulse immediately after the light pulse appeared. The influence of the charge storage, making the height of the photon pulses dependent on preceding pulses, is overcome by giving the height and duration of the applied current pulses such values that the electron and photon densities return to the start values at the end of the pulse. This makes the start condition identical for each pulse.

Numerical solutions show that the detailed current pulse form is not significant. Only the charge delivered by the current has to fulfill some requirements. Analytical formulas, derived for square current pulses, are therefore at least qualitatively applicable for a wide range of pulse forms.

The following analysis is based on the rate equations including spontaneous emission as reported in [5],

$$
\begin{aligned}
& d n / d t=J /(e d)-n / \tau_{s}-g S \\
& d S / d t=g S-S / \tau_{p}+\beta n / \tau_{s}
\end{aligned}
$$

where

$$
\begin{array}{ll}
n, J, S & \text { electron, current, and photon densities, respectively; } \\
d & \text { active layer thickness; } \\
e & \text { electronic charge; } \\
\tau_{s}, \tau_{p} & \text { electron and photon lifetimes. }
\end{array}
$$

The gain of the laser is $g=\alpha\left(n-N_{0}\right)$ [5], [6], where $N_{0}$ and $\alpha$ are constants. The factor $\beta$ is the fraction of spontaneous light that goes into the the lasing modes. In [7] it has been shown

Manuscript received October 20, 1975; revised July 1, 1976.

The author is with the Electromagnetics Institute, Technical University of Denmark, Lyngby, Denmark. that the spectrum of a narrow pulse is determined by the dc bias, and is almost independent of the current pulse height. Therefore $\beta$ is taken to depend on the prebias current only and to be constant during a pulse. The value of $\beta$ is estimated to be of the order of $10^{-4}$ when one mode is active, and is taken to be proportional to the number of active modes [8] .

For simplicity, effects like mode locking, mode coupling, $Q$ switching, electron diffusion, and circuit parasitics have been disregarded in this analysis.

\section{The Modulation Form}

In the present modulation form, the laser is prebiased by a dc current density $J=J_{0}$ near threshold. This gives the start values $n=n_{0}$ and $S=S_{0}=x S_{\mathrm{th}}$, where $S_{\mathrm{th}}$ is the static photon density at threshold and $x$ is a parameter used to describe the starting condition.

A current pulse $\Delta J(t)$, sufficiently high to give ringings [5], is superposed on $J_{0}$. The electron density $n$ now describes an oscillating curve with a maximum and a minimum (Fig. 1). The photon density reaches a maximum $S_{\max }$ when $n$ in its negative slope region has decreased to $n=n_{c} \cong 1 /\left(\alpha \tau_{P}^{\prime}\right)$ where $\tau_{p}^{\prime}=\tau_{p} /\left(1+\alpha N_{0} \tau_{p}\right)$. If the current has only small relative changes during the output of light, the pulse is nearly symmetric [5], and we have $n_{\max }-n_{c} \simeq n_{c}-n_{\min }$. After the minimum, the last part of the current pulse pumps $n$ up to $n_{0}$ after which the pulse is switched off. From this moment $S$ decays from a value only slightly higher than $S_{0}$ within $10-100 \mathrm{ps}$ to this value. Another timing of the current would give the laser a longer relaxation time, up to $4 \mathrm{~ns}$, due to the electron life time.

The necessary and sufficient conditions for the use of the present modulation model is expressed by the two following integrals of the current pulse $\Delta J(t)=J(t)-e d n_{c} / \tau_{s}$ :

$$
\begin{aligned}
& n_{\max }-n_{0}=1 / e d \int_{\text {start }}^{t\left(n=n_{\max }\right)} \Delta J d t \\
& n_{0}-n_{\min }=1 / e d \int_{t\left(n=n_{\min }\right)}^{\mathrm{end}} \Delta J d t .
\end{aligned}
$$

These integrals follow directly from (1) and the fact that before and after the light pulse has emerged, we can neglect the term $g S$. Equation (3a) expresses the pumping of the electrons by the pulse necessary to give the wanted light output pulse, while (3b) expresses the pump necessary to bring the electron density back to its start value. This charge condition shows that the detailed current pulse form is not significant for the applicability of the present model. 


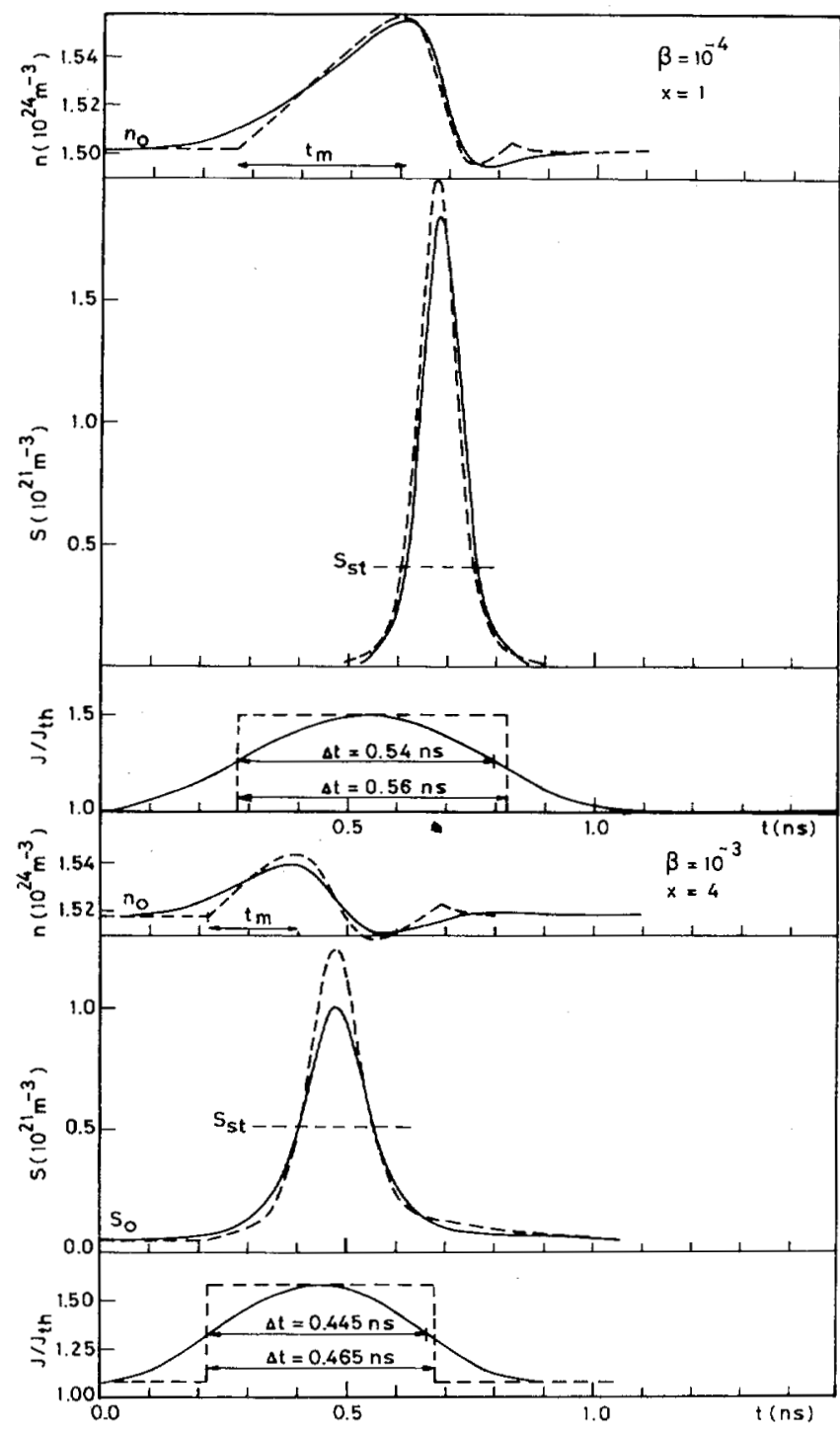

Fig. 1. Numierical results of electron and photon densities as a function of time in a pulse, calculated for a raised cosine (-) and a square (--) current pulse. The applied parameters were $\tau_{p}=2.94 /(1+$ $\left.2.67 \cdot 10^{-25} n(t)\right) \mathrm{ps}, \tau_{s}=4.0 \mathrm{~ns}, \alpha=1.125 \cdot 10^{-12} \mathrm{~s}^{-1} \cdot \mathrm{m}^{3}, N_{0}=$ $0.11 \cdot 10^{25} \mathrm{~m}^{-3}, d=0.5 \mu \mathrm{m}, e=1.6 \cdot 10^{19}$ As.

\section{NuMERICAL SOLUTION}

Fig. 1 shows computer calculations of electron and photon densities for a square and a raised cosine pulse form. Both have the same amplitude. The half-value widths $\Delta t$ of the current pulses are in both cases fitted to values according to the model. It is seen that $\Delta t$, and therefore also the charge in the pulse, is approximate independent of the current pulse form. This has been shown to be true for several sets of parameters $\beta, x$. In Fig. 1 two examples are shown where $\beta=10^{-4}, x=1$ and $\beta=10^{-3}, x=4$.

For low $\beta$ and $x$, i.e., low start values $S_{0}$, the peak and width of the light pulse are nearly independent of the current pulse shape. For higher $\beta$ and $x$ the tendency is that the maximum decreases and the width increases, but in all cases the integrated light output is the same.

Calculations of a long row of pulses showed that no intersymbol interference was present when the correct timing of the current pulses was used, independent of the current pulse spacing down to $0.1 \mathrm{~ns}$. Overtiming of the pulses gave strong intersymbol interference, while undertimed pulses with a deviation up to 10 percent gave stable light output pulses with less than 5 percent change in $S_{\max }$.

\section{ANALytical Approximations}

As a consequence of the fact that the laser response is only weakly dependent on the detailed current pulse form, and for simplicity, a square current pulse has been analyzed in order to obtain simple approximate formulas to describe the light output and the timing of the current pulse.

The computer calculations show that a square current pulse with the amplitude $\Delta J$ causes $n$ to vary approximately as a triangular wave with time. From the start to $n=n_{\max }$ and from $n=n_{\min }$ to the end of the pulse, the slope is $\Delta J^{\prime}=$ $\Delta J /(e d)$. Between $n=n_{\max }$ and $n=n_{\text {min }}$, the slope was taken to be a constant $-n^{\prime}$. Apparently rough, this approximation gives results which are in 5-10-percent agreement with computer calculations for all reasonable values of parameters $\Delta J / J_{\text {th }}<2,10^{-4}<\beta<5 \cdot 10^{-3}$, and $1<x<10$. As reference for the start condition for the pulse modulation we use the threshold values. From the definition of the threshold current density in [5] we obtain $J_{\text {th }}^{\prime}=J_{\text {th }} /(e d) \simeq 1 /\left(\alpha \tau_{s} \tau_{p}^{\prime}\right)$. The static photon and electron densities with $J^{\prime}=J_{\text {th }}^{\prime}$ are given by

$$
S_{\mathrm{th}} \cong 1 /\left(\alpha \tau_{s}\right) \cdot\left(\beta \tau_{p} / \tau_{p}^{\prime}\right)^{1 / 2}
$$

and

$$
\Delta n_{\mathrm{th}}=n_{\mathrm{th}}-n_{c} \simeq-1 / \alpha \cdot\left(\beta / \tau_{p} / \tau_{p}^{\prime}\right)^{1 / 2}
$$

where $n_{c}$ is the electron density for which the gain exceeds the total photon loss in the laser. By introduction of $\Delta n=n-n_{c}$ in (2) we obtain

$$
d S / d t=\alpha \Delta n S+\beta n / \tau_{s}
$$

The start condition is defined by $J=J_{0}$ giving $S=S_{0}=$ $x \cdot S_{\text {th }}$ and according to (5) $\Delta n=\Delta n_{0}=\Delta n_{\mathrm{th}} / x$. The analysis is now performed in three steps.

1) In the time interval $0<t<t_{m}$, where $t_{m}$ is defined by $n\left(t_{m}\right)=n_{\max }$, an approximate solution of (5) can be written as

$$
\begin{aligned}
S= & \left\{S_{1}\left(\operatorname{erf} A\left(t-t_{0}\right)+\operatorname{erf} A t_{0}\right)+S_{0} \exp \left(-A^{2} t_{0}^{2}\right)\right\} \\
& \cdot \exp A^{2}\left(t-t_{0}\right)^{2}
\end{aligned}
$$

where $t_{0}=-\Delta n_{0} / \Delta J^{\prime}$ is the time it takes for $n$ to grow from $n_{0}$ to $n_{c}$, and

$$
S_{1}=\beta /\left(\alpha \tau_{s} \tau_{p}^{\prime}\right) \cdot \sqrt{\pi /\left(2 \alpha \Delta J^{\prime}\right)}
$$

and

$$
A=\sqrt{\alpha \Delta J^{\prime} / 2} .
$$

At $t=t_{m}$ we have $d n / d t=0$. With $n_{\max } \approx n_{c}$ in (1) we find that $S \cong S_{\mathrm{st}}$, the stationary state value corresponding to $J=J_{0}+\Delta J$. This, inserted into (6), gives in practical cases, when $A\left(t_{m}-t_{0}\right) \geqslant 1$,

$$
t_{m}=t_{0}+\sqrt{2 B /\left(\alpha \Delta J^{\prime}\right)}
$$


where

$$
B=\ln \left(S_{\mathrm{st}} / S_{00}\right)
$$

and

$$
S_{00}=S_{1}\left(1+\operatorname{erf} A t_{0}\right)+S_{0} \exp \left(-A^{2} t_{0}^{2}\right) .
$$

For pulses where $\tau_{P} / \tau_{s} \cdot x^{2} / \beta \cdot \Delta J^{\prime} / J_{\text {th }}^{\prime}>1$, we find that

$$
B \simeq \ln \left(\Delta J^{\prime} / J_{\text {th }} \cdot x^{-1} \cdot\left(\tau_{p} / \tau_{p}^{\prime} / \beta\right)^{1 / 2}\right) .
$$

A limiting condition for which the model can be applied is given by $n_{\max }-n_{c}=n_{c}-n_{\min }$ and $n_{0}>n_{\min }$, which gives $t_{m} \geqslant 2 t_{0}$. By insertion of $t=2 t_{0} \leqslant t_{m}$ into the right-hand side and $S=S_{\text {st }}$ into the left-hand side of (6), we obtain, after some calculations,

$$
\Delta J / J_{\mathrm{th}} \geqslant\left(4 \tau_{s}^{2} \tau_{p}^{\prime} \cdot \tau_{p}^{-3}\right)^{1 / 4} \cdot \beta^{3 / 4} / x^{1 / 2}+\frac{1}{2} \tau_{s} / \tau_{p} \cdot \beta / x^{2}
$$

which is in 5-percent agreement with computer simulations for all practical cases, $x>1$ and $\beta<5 \cdot 10^{-3}$.

2) In the time interval $t_{m}<t<t_{\min }$, where $t_{\min }$ is defined by $n\left(t_{\min }\right)=n_{\min }$, we use $\Delta n=-n^{\prime} \Delta t^{\prime}$. Here $\Delta t^{\prime}=t-t_{m}$ $\Delta n_{\max } / n^{\prime}$ and $\Delta n_{\max }=\left(t_{m}-t_{0}\right) \Delta J^{\prime}$. Equation (5) now gives

$$
\begin{aligned}
S & =S_{\max } \exp \left(-\left(\alpha n^{\prime} / 2\right) \Delta t^{\prime 2}\right) \\
S_{\max } & =\tau_{p} \Delta J^{\prime} \exp \left(\alpha \Delta n_{\max }^{2} /\left(2 n^{\prime}\right)\right) .
\end{aligned}
$$

The integrated photon output of one pulse is given by [5]

$$
S_{\text {out }, \text { pulse }}=\frac{1}{\tau_{p}} \int_{t_{m}}^{t_{\min }} S d t=2 \Delta n_{\max }+\Delta J \cdot \Delta t_{\mathrm{st}}
$$

where $\Delta t_{\mathrm{st}}=2 \Delta n_{\text {max }} / n^{\prime}$ is the pulsewidth defined by points where $S=S_{\text {st }}$. From (12)-(14) an approximate proportionality between $n^{\prime}$ and $\Delta J^{\prime}$ ( $B$ varies slowly) is found:

$$
n^{\prime} / \Delta J^{\prime}=2 B / \ln (16 B / \pi) \text {. }
$$

Finally we can use (15) to obtain solutions for the pulse parameters

$$
\begin{aligned}
& S_{\max }=\tau_{p} \Delta J^{\prime} \cdot \sqrt{16 B / \pi} \\
& \Delta t_{\text {st }}=\sqrt{2 /\left(\alpha \Delta J^{\prime}\right)} \ln (16 B / \pi) / \sqrt{B}
\end{aligned}
$$

and the half-power width

$$
\begin{aligned}
\Delta t_{1 / 2} & =2\left(2 \ln 2 / \alpha / n^{\prime}\right)^{1 / 2} \\
& =\sqrt{2 /\left(\alpha \Delta J^{\prime}\right)}(2 \ln 2 \cdot \ln (16 B / \pi) / B)^{1 / 2} .
\end{aligned}
$$

3) In the time interval $t_{\min }<t<\Delta t$, where $\Delta t$ is the current pulse length, we can approximate the slope of $n$ by $\Delta J^{\prime}$. The value of $\Delta t$ for which $n(\Delta t)=n_{0}$ is

$$
\begin{aligned}
\Delta t & =2 \Delta n_{\max }\left(1 / \Delta J^{\prime}+1 / n^{\prime}\right) \\
& =\sqrt{2 / \alpha \Delta J^{\prime}} \cdot(2+\ln (16 B / \pi) / B) B^{1 / 2}
\end{aligned}
$$

where we once more have used (15).

The total photon output in the pulse defined by (14) is now found by

$$
S_{\text {out }, \text { pulse }}=\Delta J^{\prime} \cdot \Delta t \propto \sqrt{\Delta J^{\prime}} .
$$

The essence of the last statement is that the linear variation of $n$ leads to the result that no photon is present before and after the moments where $S=S_{\mathrm{st}}$. The error introduced in this way is less than 20 percent, which is not serious because almost all of the 20 percent is concentrated as exponential tails at both sides of the pulse.

\section{CONCLUSION}

We can conclude that the formula for $S_{\text {out, pulse agreed }}$ within 20 percent, and all other formulas within 10 percent, with computer simulation for the parameters $\Delta J / J_{0}<2$. $10^{-4}<\beta<5 \cdot 10^{-3}$ and $1<x<10$. The variation of the times is approximately inversely proportional to $\sqrt{\Delta J^{\prime}}$, while the photon pulse maximum and the integrated pulse are approximately proportional to $\Delta J^{\prime}$ and $\sqrt{\Delta J}$, respectively. These features follow from the fact that the functions of $B$ appearing as factors in the pulse parameters vary slowly with $\Delta J^{\prime}$. The only influence of $\beta$ and $x$ is also through $B[(9)$ and (10)], which is why this influence is rather weak. The tendency is that for increasing $\beta$ and $x$, the values of $t_{m}, \Delta t$, $S_{\max }$, and $S_{\text {out, pulse }}$ decrease while $\Delta t_{1 / 2}$ increases. Numerical calculation shows that in all cases $\Delta t_{1 / 2}<0.5 \Delta t$. This gives good separation between the light pulses.

Correctly timed and undertimed current pulses with pulse spacings down to $0.1 \mathrm{~ns}$ gave no or small intersymbol interference, while overtimed current pulses gave instability and strong intersymbol interference. This suggests that the model is useful for high-speed modulation. It can also be concluded that this modulation form is most useful when the prebias is a little above threshold $(x>1)$ both because of the dynamic behavior $[(11)]$ and because the spectrum then is narrow according to [7] ( $\beta$ is small). It is expected that with this model, pulse durations of $\Delta t=0.25-0.3$ ns corresponding to bit rates 3-4 Gbit/s can be obtained with $\Delta J / J_{\mathrm{th}}=1.5$ which is twice the highest experimentally obtained [9]. It is believed that adjustments of the parameters $\Delta t, \Delta J$, and $x$ to values which fit with the results of the present model have played a role in obtaining Gbit/s modulation rates in earlier experimental works. The problem of obtaining narrow current pulses $(\Delta t=300 \mathrm{ps}) \mathrm{can}$, for example, be overcome by using a MESFET driver for the laser [10].

Independently of this work some experimental work has recently been performed which verifies some features of the described modulation method [11].

\section{ACKNOWLEDGMENT}

The author wishes to thank Dr. M. T. Vlaardingerbroek and Dr. P. Jeppesen for many fruitful discussions.

\section{REFERENCES}

[1] T. P. Lee and R. M. Drosier, "Charge storage in injection lasers and its effects on high-speed pulse modulation of laser diode," Proc. IEEE, vol. 62, pp. 1176-1177, 1974.

[2] T. Ikegami, K. Kobayashi, and Y. Suematsu, "Transient behaviour of semiconductor lasers," Electr. Comm. Japan, vol. 53 B, pp. 82-89, 1970.

[3] T. Ozeki and T. Ito, "A new method for reducing pattern effects in PCM current modulation of DH-GaAlAs lasers," IEEE $J$. Quantum Electron., vol. QE-9, pp. 532-533, 1973. 
[4] M. J. Adams, "Rate equations and transient phenomena in semiconductor lasers," Opto. Electr., vol. 5, pp. 201-215, 1973.

[5] . P. M. Boers, M. Danielsen, and M. T. Vlaardingerbroek, "Dynamic behaviour of semiconductor lasers," Electr. Lett., vol. 11, pp. 206-208, May 1975.

[6] B. W. Hakki and L. Paoli, "C. W. degradation of $300 \mathrm{~K}$ of GaAs double heterostructure junction lasers II," J. Appl. Phys., vol. 44, pp. 4113-4119, 1973.

[7] P. R. Selway and A. R. Goodwin, "Effects of D. C. bias level on the spectrum of GaAs lasers operated with short pulses," Elect. Lett., vol. 12, pp. 25-26, 1976.
[8] D. Röss, Laser, Light Amplifiers and Oscillators. New York: Academic p. 284, 1969.

[9] P. Russer and S. Schultz, "Direkte Modulation eines Doppelheterostrukturlasers mit einer bitrate von $2.3 \mathrm{Gbit} / \mathrm{s}, "$ A.E.U., vol. 27, pp. 193-194, 1973.

[10] V. Ostoich, N. Slaymaker, and P. Jeppesen, "Gbit/s modulation of DH-GaAlAs lasers with GaAs MESFET's," presented at the 1st European Conf. Opt. Fibre Comm., Sept. 1975.

[11] J. G. Farrington and J. E. Carroll, "Sub-nanosecond pulsing of GaAs stripe lasers," presented at the 1st European Conf. Opt. Fibre Comm., Sept. 1975.

\title{
Zero-Power-Gain Measurements in CW HF(DF) Laser by Means of a Fast Scan Technique
}

\author{
RICHARD A. CHODZKO, DONALD J. SPENCER, HAROLD MIRELS, STEPHEN B. MASON, AND DAVID H. ROSS
}

\begin{abstract}
An improved technique for measuring zero power gain in a CW HF-DF chemical laser has been developed in which a CW HF(DF) single-line, frequency-stabilized TEM $_{00}$ mode probe laser is used. Through use of a flat rotating mirror and focusing elements, the streamwise distribution of zero-power gain was scanned at a rate of $\sim 1 \mathrm{~mm} / \mu$ s with a spatial resolution of $\sim 1 \mathrm{~mm}$. The zero-power gain profile was observed for two arc-driven chemical-laser nozzles with both HF and DF active species. The nozzles consisted of a 36-slit array with perforated tube $\mathrm{H}_{2}$ injectors and a 55-slit array with uniform $\mathrm{H}_{2}$ injection. Results are presented for the variation of zero power gain with axial distance, the magnitude and location of peak gain, and the gain cutoff location for a number of transitions. Peak gain values of 15 percent $/ \mathrm{cm}$ and 5.5 percent $/ \mathrm{cm}$ were measured with HF and DF active species, respectively, with the 55 -slit nozzle array.
\end{abstract}

\section{INTRODUCTION}

S MALL-SIGNAL-GAIN measurements in an arc-driven, supersonic diffusion CW HF chemical laser have been previously made both directly by means of pulsed-probe laser [1] and indirectly by means of a chemiluminescence technique [2]. The measurements made with a pulsed-probe laser had an uncertainty in the magnitude of the gain that resulted from a lack of frequency stability in the multitransverse, multilongitudinal mode probe beam. The gain variation with distance from the nozzle exit plane $x$ was obtained [1] by measuring the gain on the various HF vibrational rotational transitions at fixed values of $x$ during a given arc run. The arc

Manuscript received December 10, 1975; revised July 1, 1976. This work was supported by the Air Force Weapons Laboratory under U.S. Air Force Space and Missile Systems Organization (SAMS) Contract F04701-75-0076.

The authors are with The Aerospace Corporation, El Segundo, CA 90245 . was then shut down, the probe beam was located at a new value of $x$, and the process repeated.

Clearly, real-time (fast) scans with a CW HF(DF) probe would be desirable in order to reduce run-to-run flow variations and to expedite measurements. The present work incorporates a newly developed single-transverse, singlelongitudinal mode, stable $\mathrm{CW}$ probe laser and an optical system that permits scanning of the small-signal CW HF(DF) gain profile from the nozzle exit plane to a distance $8 \mathrm{~cm}$ downstream in about $0.3 \mathrm{~ms}$ with a $1-\mathrm{mm}$ spatial resolution. Zero-power-gain distributions have been obtained by means of two different nozzle configurations with both $\mathrm{HF}$ and $\mathrm{DF}$ active species. The results are reported herein.

\section{Experimental Apparatus and Method}

\section{A. Scanning Technique}

The gain profile scanning technique used in the present experiments is shown in Fig. 1. A technique similar to this was used previously by Falk [3] for zero-power-gain measurements in a $\mathrm{DF}-\mathrm{CO}_{2}$ transfer chemical laser. The rotating mirror was located at the focal points of the focusing elements $L_{2}$ (3-m radius concave mirrors), which caused the probe beam to sweep from the nozzle exit plane to a distance $8 \mathrm{~cm}$ downstream while remaining parallel to the exit plane. The IR probe beam was aligned with a HeNe reference beam to the center line (vertical) of the nozzle exit. The focusing element $L_{3}$ then imaged the split probe beams on diffuse reflectors (sandblasted $\mathrm{Al}$ plates) viewed by the signal and reference detectors. The signal detector voltage was observed versus time with $\mathrm{H}_{2} / \mathrm{D}_{2}$ off and on. The $\log$ of the ratio of these observations defines the variation of active region gain versus 